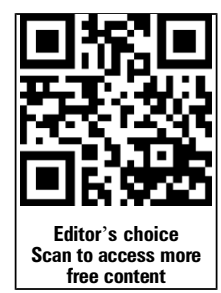

${ }^{1}$ Pediatric Emergency Department, Geneva University Hospital, Geneva, Switzerland ${ }^{2}$ Pediatric Emergency Department, University Children's Hospital,

Zurich, Switzerland

${ }^{3}$ Department of Diagnostic Imaging, University Children's Hospital, Zürich, Switzerland ${ }^{4}$ Department of Pediatric Surgery, Ostschweizer Children's Hospital, St. Gallen, Switzerland

${ }^{5}$ Division of Clinical Chemistry and Biochemistry, University Children's Hospital, Zurich,

Switzerland

${ }^{6}$ Pediatric Surgery Department, Geneva University Hospital, Geneva, Switzerland

${ }^{7}$ Pediatric Surgery Department, University Children's Hospital, Zurich, Switzerland

\section{Correspondence to}

Dr Sergio Manzano, Pediatric Emergency Department,

Geneva University Hospital, 6, rue Willy-Donze, Geneva 1211 Switzerland;

sergio.manzano@hcuge.ch

Received 14 November 2014 Revised 20 July 2015 Accepted 28 July 2015 Published Online First 17 August 2015

\title{
Diagnostic performance of S100B protein serum measurement in detecting intracranial injury in children with mild head trauma
}

\author{
Sergio Manzano, ${ }^{1}$ Iris Bachmann Holzinger, ${ }^{2}$ Christian J Kellenberger, ${ }^{3}$ \\ Laurence Lacroix, ${ }^{1}$ Dagmar Klima-Lange, ${ }^{4}$ Martin Hersberger, ${ }^{5}$ Giorgio La Scala, ${ }^{6}$ \\ Stefan Altermatt, ${ }^{7}$ Georg Staubli ${ }^{2}$
}

\begin{abstract}
Objective To assess the accuracy of S100B serum level to detect intracranial injury in children with mild traumatic brain injury.

Methods A multicenter prospective cohort study was carried out in the paediatric emergency departments of three tertiary hospitals in Switzerland between January 2009 and December 2011. Participants included children aged $<16$ years with a mild traumatic brain injury (GCS $\geq 13$ ) for whom a head CT was requested by the attending physician. Venous blood was obtained within $6 \mathrm{~h}$ of the trauma in all children for S100B measurement before a head CT was performed. As the S100B value was not available during the acute care period, the patient's management was not altered. The main measures were protein S100B value and the CT result.

Results $20 / 73$ (27.4\%) included children had an intracranial injury detected on CT. S100B receiver operating characteristics area under the curve was 0.73 (95\% Cl 0.60 to 0.86). With a $0.14 \mu \mathrm{g} / \mathrm{L}$ cut-off point, S100B reached an excellent sensitivity of $95 \%(95 \% \mathrm{Cl}$ $77 \%$ to $100 \%)$ and $100 \%(95 \% \mathrm{Cl} 81 \%$ to $100 \%)$ in all children and in children aged $>2$ years, respectively.

\section{Key messages}

What is already known about this subject?

- Studies in adults show that serum S100B is a possible adjunct to clinical decision rules to detect intracranial injury, and the American College of Emergency Physicians suggests that S100B measurement could reduce the number of unnecessary CTs.

- Research in children on this biomarker is limited and it shows interesting but conflicting results.

What does this study add?

- In this multicentre, prospective cohort study in patients aged $<16$ years, S100B was found to have excellent sensitivity, making it an accurate tool to rule out intracranial injuries. Its poor specificity is a limiting factor that precludes its use as the sole marker.

- Used with clinical decision rules, S100B may help to reduce the number of unnecessary CTs in children.
\end{abstract} The specificity, however, was 34\% (95\% Cl 27\% to $36 \%$ ) and $37 \%(95 \% \mathrm{Cl} 30 \%$ to $37 \%$ ), respectively. Conclusions $\mathrm{S100B}$ has an excellent sensitivity but poor specificity. It is therefore an accurate tool to help rule out an intracranial injury but cannot be used as the sole marker owing to its specificity. Used with clinical decision rules, S100B may help to reduce the number of unnecessary CT scans.

\section{INTRODUCTION}

Traumatic brain injury (TBI) is a very frequent cause of presentation to the emergency department. This condition accounts in the USA for more than 500000 visits a year in children. ${ }^{1}$ The vast majority of children with mild TBI, defined as GCS $13-15$, $^{2}$ have no intracranial injury (ICI): ${ }^{3}$ a lesion is seen in $3-7 \%$ of these children undergoing $\mathrm{CT}^{3-6}$ and only $0.1-0.6 \%$ need a neurosurgical intervention. ${ }^{3} 5$

Consequences of a missed ICI may be devastating and its symptoms or signs, such as vomiting, headache or amnesia, are often misleading. CT is therefore performed in almost $50 \%$ of children with mild $\mathrm{TBI}^{3}$ as this is the only diagnostic tool to detect an ICI in the emergency setting.
However, overuse of CT scans has been called into question because the ionising radiation can lead to lethal malignancies. This rate can be as high as 1 in 1000 head CT scans, with younger children being more susceptible. ${ }^{7}$

Several decision rules based on clinical signs have been described to reduce the number of unnecessary CT scans.. ${ }^{3} 5910$ Although these guidelines have excellent sensitivity and good specificity, they are not widely used, ${ }^{11}$ and a sharp increase in the use of CT has been noted. ${ }^{12} 13$ Groups at very low risk for ICI have been identified. However, for all other children, the decision to obtain a CT scan is left to the physician and often based on his/her own experience. ${ }^{3}$

$\mathrm{S} 100 \mathrm{~B}$ is a calcium-binding protein which is located predominantly in the cytoplasm and nucleus of astrocytes and Schwann cells. ${ }^{14}$ It is released by damaged cells and enters the systemic circulation only when the blood-brain barrier is disrupted. It is then excreted in urine within $6 \mathrm{~h}$ from injury. ${ }^{15} \mathrm{~S} 100 \mathrm{~B}$ is also expressed to a lesser extent in adipocytes and chondrocytes, and thus long-bone fractures may increase the systemic S100B level even in the absence of brain injury. ${ }^{16}$
Kellenberger $\mathrm{Cl}$, et al.

Emerg Med J 2016;33:

42-46. 
Many adult studies have shown that serum S100B is a possible adjunct to clinical decision rules to detect ICI, ${ }^{17-21}$ with the American College of Emergency Physicians suggesting that S100B measurement could reduce the number of unnecessary CT scans by $30 \% .^{22}$

A few studies have evaluated $\mathrm{S} 100 \mathrm{~B}$ as a tool to reduce the number of CT scans in children, with interesting but conflicting results. $^{23-27}$

The objective of our study was to assess the accuracy of S100B serum level to detect ICI in children with mild TBI.

\section{METHODS}

Study design

This was a multicentre prospective cohort study. The study received institutional review board approval of each participating hospital and was conducted in accordance with Good Clinical Practice guidelines and provisions of the Declaration of Helsinki.

\section{Settings and selection of participants}

The study was conducted in the paediatric emergency departments of three tertiary hospitals in Switzerland on a consecutive sample of patients between January 2009 and December 2011. These three centres see 33000,24000 and 12000 patients a year. We included all children aged $<16$ years with a mild TBI (GCS $\geq 13$ ) for whom a head CT was requested by the attending physician. We excluded those children who arrived at the hospital more than $6 \mathrm{~h}$ after the trauma, children with Down syndrome (since in these patients $\mathrm{S} 100 \mathrm{~B}$ is overexpressed ${ }^{28}$ ) or patients with a history of convulsion in the past 7 days.

\section{Study protocol}

The attending paediatric emergency physicians obtained consent from the parents of children meeting the inclusion criteria for participation in the study. After consent was obtained, venous blood was drawn and centrifuged. The serum was then frozen $\left(-20^{\circ} \mathrm{C}\right)$ and sent in batches to the division of clinical chemistry and biochemistry of the University Children's Hospital Zurich for S100B measurement. As the S100B value was not available during the acute care period, the patient's management was not altered.

All head CT scans were reviewed for traumatic injuries by one paediatric radiologist (CJK), who was blinded to the patient's clinical signs and the $\mathrm{S} 100 \mathrm{~B}$ value.

\section{S100B measurement}

All S100B ( $\alpha \alpha$ and $\alpha \beta$ dimers) measurements were done on an Elecsys 2010 using the commercial S100 assay from Roche (Roche Diagnostics, Rotkreuz, Switzerland), with an interassay coefficient of variation $<2.8 \%$ according to the manufacturer.

\section{Outcome measures}

Primary outcome was evaluation of the diagnostic value of $\mathrm{S} 100 \mathrm{~B}$ in detecting intracranial injuries in children aged $<16$ years with mild head trauma. Secondary outcomes were evaluation of the same parameters excluding children aged $<2$ years (age group where normal S100B values vary physiologically with age) and comparison of signs and symptoms between the groups with and without ICI (all children).

\section{Definitions}

Mild TBI: acute head trauma with a GCS 13-15, with confusion or loss of consciousness $(<30 \mathrm{~min})$ or amnesia or transient neurological abnormality. ${ }^{2}$
ICI: any collection of blood within the cranial vault or cerebral oedema.

\section{Data analysis and sample size}

A $\chi^{2}$ test was used to describe categorical data. A t test was used to compare continuous variables as normality was observed in our data. The $95 \%$ CIs of the results are reported. A level of $\mathrm{p}<0.05$ was considered significant. Statistical analyses were performed using PASW V.18 (SPSS Inc, Chicago, USA).

\section{RESULTS}

Between January 2009 and December 2011, 2595 children were admitted for a mild TBI and $342(13.2 \%)$ had a head CT scan. A total of 80 children with mild TBI meeting the inclusion criteria were enrolled (see figure 1). Seven patients were excluded (no S100B measurement: three patients; venous puncture $>6$ h after trauma: one patient; no CT performed: three patients). Of the 73 included children, 20 (27.4\%) had an ICI detected on CT. The lesion was an epidural haematoma in nine children, a subarachnoid haemorrhage in four, an epidural haematoma and subarachnoid haemorrhage in three. The remaining four children had respectively a subdural haematoma, an epidural and subdural haematoma, a subdural haematoma and subarachnoid haemorrhage and haemorrhagic parenchymal contusion. No surgical intervention was required. There was no statistically significant difference between the patients with or without ICI in age, gender and time between trauma and venous puncture for S100B measurement (table 1).

Clinical features that might influence a physician's decision to obtain a CT scan are shown and compared between groups in table 1 . The only statistically significant difference between groups was the S100B value.

The S100B receiver operating characteristics (ROC) curve is shown in figure 2. The area under the curve was $0.73(95 \% \mathrm{CI}$ 0.60 to 0.86$)$. After excluding children aged $<2$ years, the area under the curve was 0.77 (95\% CI 0.65 to 0.89 ) for the remaining 64 children.

Using the best cut-off value derived from the ROC analysis $(0.14 \mu \mathrm{g} / \mathrm{L}), \mathrm{S} 100 \mathrm{~B}$ reached a sensitivity of $95 \%(19 / 20)(95 \%$ CI $77 \%$ to $100 \%)$ and $100 \%(18 / 18)(95 \%$ CI $81 \%$ to $100 \%)$ in all children and in children aged $>2$ years, respectively. With this cut-off point, one (1.4\%) child (aged 17 months) with a $1.5 \mathrm{~cm}$ temporoparietal epidural haematoma would have been missed. The corresponding specificity and the derived positive and negative likelihood ratio for all children and children aged $>2$ years are shown in table 2. This cut-off point has been chosen to take into account the best sensitivity/specificity ratio. If the aim was $100 \%$ sensitivity, specificity would fall to $4 \%$ (with a $0.07 \mu \mathrm{g} / \mathrm{L}$ cut-off value).

\section{DISCUSSION}

This study shows that S100B protein has good diagnostic properties with an area under the curve of 0.73 and, with $0.14 \mu \mathrm{g} / \mathrm{L}$ cut-off value, a very good sensitivity but poor specificity, in children aged $<16$ years with mild TBI (GCS 13-15).

We evaluated the same parameters after exclusion of children aged <2-years. As expected these are slightly better: the area under the curve rises to 0.77 and sensitivity reaches $100 \%$ with similar specificity. The reason for this is probably because the S100B value is age-dependent with physiologically higher levels in the youngest subjects. ${ }^{29-31}$ Reference value studies mainly use two age groups: under or over 2-3 years of age. ${ }^{29-31}$

Taking S100B as the only identifier of ICI, 18 (24.7\%) head CT scans would have been avoided. However, one $(1.4 \%)$ child 
Figure 1 S100B cut-off value $0.14 \mu \mathrm{g} / \mathrm{L}$. ICl, Intracranial injury.

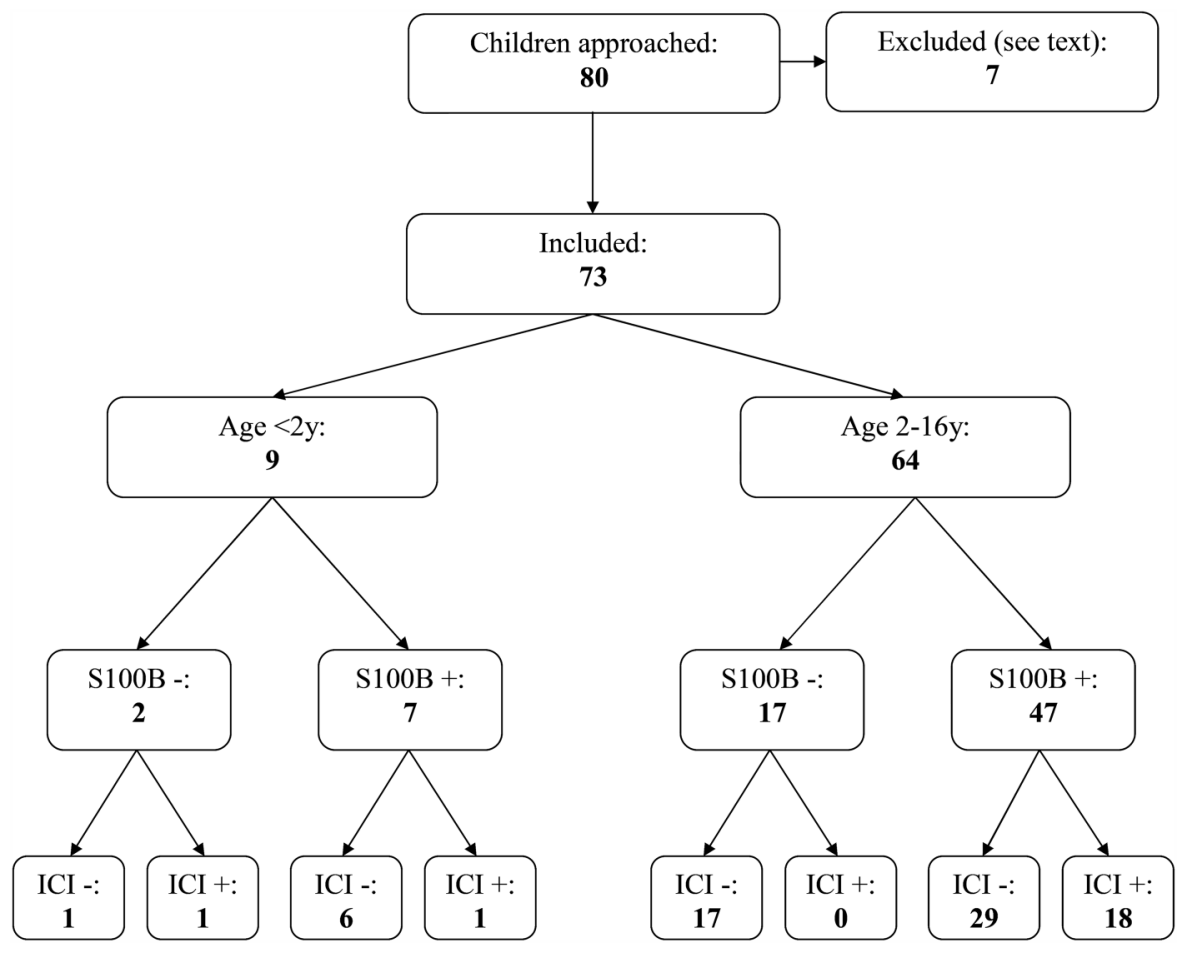

with a $1.5 \mathrm{~cm}$ epidural haematoma would have been missed. Thus, the decision to perform a CT scan cannot rely on the biomarker alone. In order to reduce unnecessary CT scans and radiation-induced malignancies, several decision rules based on clinical signs have been described. ${ }^{35910}$ The strongest validated rule to date published by Kuppermann $e t ~ a l^{3}$ suggests obtaining a CT scan in all children with GCS $<15$ or other signs of altered mental status or signs of basilar skull fracture, but leaves the decision to the physician if the child has any history of loss of consciousness, vomiting, a severe mechanism of injury or severe headache. According to these authors, the decision should be based, among other factors, on the physician's experience. In our opinion, this is the situation in which S100B could help to avoid unnecessary CT.

Most evaluations of the diagnostic accuracy of S100B come from reports on adults. Unden and Romner ${ }^{20}$ included 12 studies with 2466 patients in a systematic review and

Table 1 Comparison of demographic and clinical features between the groups with and without ICI based on CT

\begin{tabular}{lllc}
\hline Group & $\begin{array}{l}\text { Without ICI } \\
(\mathrm{n}=53)\end{array}$ & $\begin{array}{l}\text { With ICI } \\
(\mathrm{n}=20)\end{array}$ & $\mathbf{p}$ Value \\
\hline Age (months), mean (SD) & $94.0(56.5)$ & $78.1(44.4)$ & 0.21 \\
Male, $\mathrm{n}(\%)$ & $35(66.0)$ & $16(80.0)$ & 0.38 \\
$\begin{array}{l}\text { Time trauma to venous puncture } \\
\text { (min), mean (SD) }\end{array}$ & $163.8(84.7)$ & $186.0(106.0)$ & 0.41 \\
GCS <15, n (\%) & $19(35.8)$ & $8(40.0)$ & 0.94 \\
Vomiting, $\mathrm{n}(\%)$ & $25(47.2)$ & $8(40.0)$ & 0.76 \\
Headache, $\mathrm{n}(\%)$ & $28(52.8)$ & $13(65.0)$ & 0.50 \\
Amnesia, $\mathrm{n}(\%)$ & $17(32.1)$ & $6(30.0)$ & 1.00 \\
Seizure, $\mathrm{n}(\%)$ & $4(7.5)$ & $2(10.0)$ & 1.00 \\
S100B value ( $\mu \mathrm{g} / \mathrm{L})$, mean (SD) & $0.35(0.45)$ & $0.97(1.29)$ & $<0.05$ \\
(range) & $(2.79)$ & $(5.46)$ & \\
\hline ICI, intracranial injury. & & &
\end{tabular}

meta-analysis. They found sensitivity and specificity results similar to ours ( $97 \%$ and $40 \%$, respectively). The largest prospective cohort comparing S100B values with CT results in 1559 adults showed the same sensitivity (98.6\%) but worse specificity $(12 \%)$; the area under the curve was $0.76 .^{21}$

Our results are also comparable to those of the few paediatric studies published. Babcock et $a l^{23}$ included children aged 0-18 years with TBI of all grades; the area under the curve was 0.71 . However, to achieve optimal sensitivity and specificity, the cut-off value was much lower than ours $(0.006 \mu \mathrm{g} / \mathrm{L})$. The reason might be a different definition of ICI, comprising skull fractures. Castellani $e t a l^{32}$ selected 109 children with only mild TBI (GCS 13-15). With a cut-off value comparable to ours $(0.16 \mu \mathrm{g} / \mathrm{L})$ and they again found similar sensitivity $(100 \%)$ and specificity $(42 \%)$; the area under the curve was 0.68 .

In contrast, Hallen et $a^{27}$ published much higher S100B diagnostic properties. The area under the curve was 0.93 , sensitivity $100 \%$ and specificity $88 \%$. The difference between their study and other studies was the definition of a negative outcome. Children were considered to be without ICI if CT was normal or the course after observation was satisfactory, with only $10 \%$ of patients having a CT scan. This is an interesting point and possibly a limitation of our study. We considered positive any intracranial abnormality, even, probably, lesions without consequences, as in previous studies. $^{21} 232432$ It is, however, difficult to recognise those with potential for deterioration. Because a S100B value is undetectable after $6 \mathrm{~h}$ from injury in $36 \%$ of patients with initially detectable levels, ${ }^{33}$ we decided to include only children within $6 \mathrm{~h}$. It is not clear whether earlier blood sampling would have altered the diagnostic properties. Bouvier et $a l^{25}$ found a $100 \%$ sensitivity and $33 \%$ specificity when including children within $3 \mathrm{~h}$ of the TBI. However, others ${ }^{27} 32$ had the same sensitivity with better specificity when including children within $6 \mathrm{~h}$.

S100B protein is expressed in astrocytes and Schwann cells, but also in chondrocytes and adipocytes. ${ }^{14}$ This raised the 
A

ROC curve

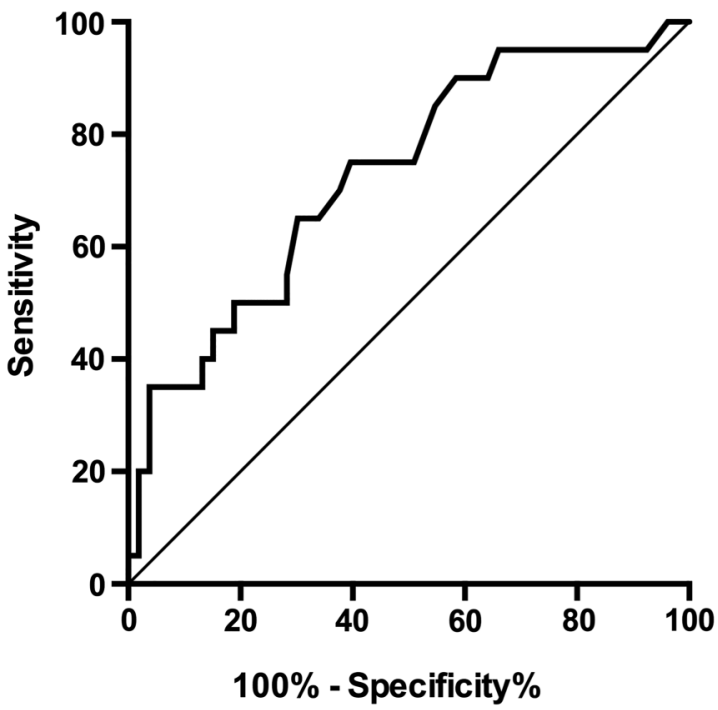

B

ROC curve

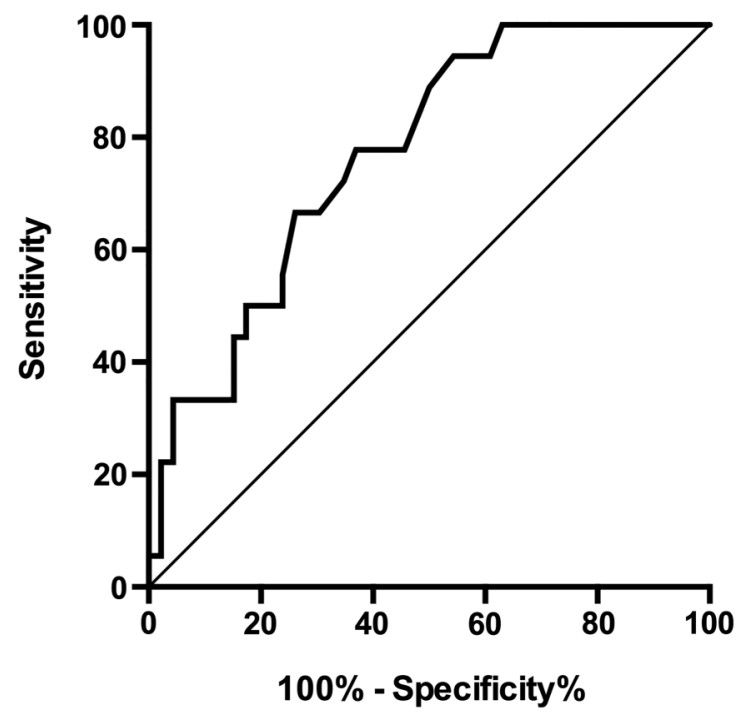

Figure 2 Receiver operating characteristic (ROC) curve for S100B to detect an intracranial injury in children aged $<16$ years (A) and in children aged 2-16 years (B) with mild head trauma.

question as to whether other lesions, such as long-bone fractures, could raise the serum S100B level, with conflicting results in the paediatric literature. Unlike Berger et al, ${ }^{34}$ who found no influence, Bechtel et $a l^{24}$ showed that children with ICI and long-bone fractures had significantly higher levels of S100B than those without a fracture, possibly affecting the specificity of the biomarker. This aspect could not be evaluated in our study because only one child had long-bone fracture (with a S100B of $1.62 \mu \mathrm{g} / \mathrm{L})$.

Another interesting finding is that we found no statistically significant difference between the groups in the presence of ICI-related worrying symptoms, whereas S100B levels were higher in the group with ICI. That point was also evaluated by Babcock et al, ${ }^{23}$ but with opposite results. Their two groups significantly differed in their responses concerning nausea or vomiting, headache and amnesia. The difference might be explained by their study population that included TBI of all severities. Also, there may be a selection bias for our patients, possibly identified as needing a CT scan based on these symptoms. Indeed, ICI was found in $27.4 \%$ of our population, compared with $17.4 \%$ in the study of Babcock et al. ${ }^{23}$

\section{Limitations}

Our study has some limitations. First, is the small sample size. Because of this, we could not draw conclusions in the subgroup aged $<2$ years and we had no surgical intervention. It would have been interesting to compare these children with those who received conservative treatment. However, despite our small sample size, we obtained results similar to those of much larger adult $^{2021}$ and paediatric studies. ${ }^{23-26} 32$ Also, the study was conducted in three tertiary hospitals, but the inclusion period and contribution were not equal, with most of the study patients coming from the two biggest centres. As stated before, our study population is limited to children undergoing head CT, with the risk of considering positive any intracranial abnormality, even, possibly, lesions without consequences. However, the benefit is to have objective outcomes, as it is difficult to identify patients at risk of deterioration.

In conclusion, the biomarker S100B is a valuable tool to help the physician decide whether head CT is indicated for children aged $<16$ years with mild head trauma. Its excellent sensitivity indicates that it could be an accurate tool to 'rule out' an ICI. However, its poor specificity is a limiting factor that precludes

Table 2 Diagnostic characteristics of S100B in children aged $<2$ years, children aged $2-16$ years and all children ( $<16$ years)

\begin{tabular}{|c|c|c|c|}
\hline S100B & Children $<2$ years & Children $2-16$ years & All children $<16$ years \\
\hline $\mathrm{n}$ total (n with ICI) & $9(2)$ & $64(18)$ & $73(20)$ \\
\hline Area under the curve & $0.57(0.00-1.00)$ & $0.77(0.65-0.89)$ & $0.73(0.60-0.86)$ \\
\hline Sensitivity $(95 \% \mathrm{Cl})^{*}$ & $50.0 \%(5 \%$ to $97 \%)$ & $100.0 \%(81 \%$ to $100 \%)$ & $95.0 \%(77 \%$ to $100 \%)$ \\
\hline Specificity $(95 \% \mathrm{Cl})^{*}$ & $14.3 \%(1 \%$ to $28 \%)$ & $37.0 \%$ (30\% to $37 \%)$ & $34.0 \%(27 \%$ to $36 \%)$ \\
\hline $\mathrm{LR}+(95 \% \mathrm{Cl})^{*}$ & $0.58(0.05$ to 1.35$)$ & $1.59(1.16$ to 1.59$)$ & $1.44(1.05$ to 1.55$)$ \\
\hline $\mathrm{LR}-(95 \% \mathrm{Cl})^{*}$ & $3.50(0.10$ to 74.19$)$ & $0.00(0.00$ to 0.63$)$ & 0.15 (0.01 to 0.84$)$ \\
\hline S100B value, mean(SD) & $0.40(0.35)$ & $0.54(0.86)$ & $0.52(0.81)$ \\
\hline Time trauma to venous puncture (min), mean (SD) & $182.1(91.3)$ & $168.2(91.3)$ & $169.9(90.8)$ \\
\hline
\end{tabular}


its use as a sole marker. Used with clinical decision rules, S100B may help to reduce the number of unnecessary CT scans.

Twitter Follow Sergio Manzano at @SwissPedEmerg

Contributors IBH, GS and SM conceived the study, designed the trial and obtained research funding. IBH and GS supervised the conduct of the trial and data collection. LL, DK-L, GLS and SA undertook recruitment of participating centres and patients and managed the data, including quality control. CJK interpreted all study CT scans. MH supervised the S100B laboratory analysis and its data. SM and LL provided statistical advice on study design and analysed the data. SM drafted the manuscript and all authors contributed substantially to its revision.

Funding Roche Switzerland supplied the S100 reagents without charge.

Competing interests None declared.

Patient consent Obtained.

Ethics approval Institutional ethics commitee (Commission Cantonale d'ethique de la recherche).

Provenance and peer review Not commissioned; externally peer reviewed.

Correction notice Since this paper was first published online figure 2 has been updated so that it displays two different ROC curves.

\section{REFERENCES}

1 Rutland-Brown W, Langlois JA, Thomas KE, et al. Incidence of traumatic brain injury in the United States, 2003. Head Trauma Rehabil 2006;21:544-8.

2 Kristman VL, Borg J, Godbolt AK, et al. Methodological issues and research recommendations for prognosis after mild traumatic brain injury: results of the International Collaboration on Mild Traumatic Brain Injury Prognosis. Arch Phys Med Rehabil 2014;95(3 Suppl):S265-77.

3 Kuppermann N, Holmes JF, Dayan PS, et al. Identification of children at very low risk of clinically-important brain injuries after head trauma: a prospective cohort study. Lancet 2009;374:1160-70.

4 Dietrich AM, Bowman MJ, Ginn-Pease ME, et al. Pediatric head injuries: can clinical factors reliably predict an abnormality on computed tomography? Ann Emerg Med 1993;22:1535-40.

5 Osmond MH, Klassen TP, Wells GA, et al. CATCH: a clinical decision rule for the use of computed tomography in children with minor head injury. CMAJ 2010;182:341-8..

6 Quayle KS, Jaffe DM, Kuppermann N, et al. Diagnostic testing for acute head injury in children: when are head computed tomography and skull radiographs indicated? Pediatrics 1997;99:E11.

7 Mathews JD, Forsythe AV, Brady Z, et al. Cancer risk in 680,000 people exposed to computed tomography scans in childhood or adolescence: data linkage study of 11 million Australians. BMJ 2013;346:f2360.

8 Pearce MS, Salotti JA, Little MP, et al. Radiation exposure from CT scans in childhood and subsequent risk of leukaemia and brain tumours: a retrospective cohort study. Lancet 2012;380:499-505.

9 Atabaki SM, Stiell IG, Bazarian JJ, et al. A clinical decision rule for cranial computed tomography in minor pediatric head trauma. Arch Pediatr Adolesc Med 2008;162:439-45.

10 Palchak MJ, Holmes JF, Vance CW, et al. A decision rule for identifying children at low risk for brain injuries after blunt head trauma. Ann Emerg Med 2003;42:492-506.

11 Stiell IG, Bennett C. Implementation of clinical decision rules in the emergency department. Acad Emerg Med 2007;14:955-9.
12 Blackwell CD, Gorelick M, Holmes JF, et al. Pediatric head trauma: changes in use of computed tomography in emergency departments in the United States over time. Ann Emerg Med 2007;49:320-4.

13 Larson DB, Johnson LW, Schnell BM, et al. Rising use of CT in child visits to the emergency department in the United States, 1995-2008. Radiology 2011;259:793-801.

14 Zimmer DB, Cornwall EH, Landar A, et al. The S100 protein family: history, function and expression. Brain Res Bull 1995;37:417-29.

15 Donato R. Intracellular and extracellular roles of $\$ 100$ proteins. Microsc Res Tech 2003:60:540-51.

16 Unden J, Bellner J, Eneroth $M$, et al. Raised serum S100B levels after acute bone fractures without cerebral injury. J Trauma 2005;58:59-61.

17 Kotlyar S, Larkin GL, Moore CL, et al. S100b immunoassay: an assessment of diagnostic utility in minor head trauma. J Emerg Med 2011;41:285-93.

18 Leidel BA, Bogner V, Zock M, et al. Serological determination of protein S100B. Significance in emergency diagnosis of adults with mild craniocerebral trauma--meta-analysis. Unfallchirurg 2012;115:903-12.

19 Muller B, Evangelopoulos DS, Bias K, et al. Can S-100B serum protein help to save cranial $\mathrm{CT}$ resources in a peripheral trauma centre? A study and consensus paper. Emerg Med J 2011;28:938-40.

20 Unden J, Romner B. Can low serum levels of S100B predict normal CT findings after minor head injury in adults? an evidence-based review and meta-analysis. J Head Trauma Rehabil 2010;25:228-40.

21 Zongo D, Ribereau-Gayon R, Masson F, et al. S100-B protein as a screening tool for the early assessment of minor head injury. Ann Emerg Med 2012;59:209-18.

22 Jagoda AS, Bazarian JJ, Bruns JJ Jr, et al. Clinical policy: neuroimaging and decisionmaking in adult mild traumatic brain injury in the acute setting. Ann Emerg Med 2008:52:714-48.

23 Babcock L, Byczkowski T, Mookerjee $S$, et al. Ability of S100B to predict severity and cranial CT results in children with TBI. Brain Inj 2012;26:1372-80.

24 Bechtel K, Frasure S, Marshall C, et al. Relationship of serum S100B levels and intracranial injury in children with closed head trauma. Pediatrics 2009;124: e697-704..

25 Bouvier D, Fournier M, Dauphin JB, et al. Serum S100B determination in the management of pediatric mild traumatic brain injury. Clin Chem 2012;58:1116-22.

26 Filippidis AS, Papadopoulos DC, Kapsalaki EZ, et al. Role of the S100B serum biomarker in the treatment of children suffering from mild traumatic brain injury. Neurosurg Focus 2010;29:E2.

27 Hallen M, Karlsson M, Carlhed R, et al. S-100B in serum and urine after traumatic head injury in children. J Trauma 2010;69:284-9.

28 Mrak RE, Griffin WS. Trisomy 21 and the brain. J Neuropathol Exp Neurol 2004;63:679-85

29 Astrand R, Romner B, Lanke J, et al. Reference values for venous and capillary S100B in children. Clin Chim Acta 2011:412:2190-3.

30 Bouvier $D$, Castellani C, Fournier $M$, et al. Reference ranges for serum S100B protein during the first three years of life. Clin Biochem 2011;44:927-9.

31 Gazzolo D, Michetti F, Bruschettini M, et al. Pediatric concentrations of S100B protein in blood: age- and sex-related changes. Clin Chem 2003:49(6 Pt 1):967-70.

32 Castellani C, Bimbashi P, Ruttenstock E, et al. Neuroprotein s-100B - a useful parameter in paediatric patients with mild traumatic brain injury? Acta Paediatr 2009;98:1607-12.

33 Ingebrigtsen $\mathrm{T}$, Waterloo $\mathrm{K}$, Jacobsen $\mathrm{EA}$, et al. Traumatic brain damage in minor head injury: relation of serum S-100 protein measurements to magnetic resonance imaging and neurobehavioral outcome. Neurosurgery 1999;45:468-75; discussion 75-6.

34 Berger RP, Pierce MC, Wisniewski SR, et al. Serum S100B concentrations are increased after closed head injury in children: a preliminary study. J Neurotrauma 2002;19:1405-9. 